\title{
SINGULARITY CONDITION OF WRIST-PARTITIONED 6-R SERIAL MANIPULATOR BASED ON GRASSMANN-CAYLEY ALGEBRA
}

\author{
Luc Djimon Clément AKONDE
}

Ph-D Student, State Key Laboratory of Digital Manufacturing Equipment and Technology, Mechanical school of Science \& Engineering, Huazhong University of Science and Technology, Wuhan, China, goering20@yahoo.fr

\begin{abstract}
To prevent the singularity of serial robot's due to the lost of one or more degree of twist freedom, it is necessary to determine the Jacobian matrix $J$ associated to its instantaneous motion and analyze the vanishing condition of the determinant det (J). Usually, the large expression of det (J) does not facilitate an efficient geometric analysis. Since Grassmann-Cayley Algebra (GCA) has powerful tools for geometric interpretation of coordinate free representation and singularity analyzing in real time computing, this method is implemented in the present work. The goal of this research is to determine the singularity condition of wristpartitioned 6-R serial manipulator (SM) based on GCA. The symbolic approach of Plücker coordinate lines is used to formulate the twist system (TS) of SM. The twist system is similar to det $(J)$ which rows are Plücker coordinate lines. The vanishing condition of det $(J)$ based on the linearity condition of TS is determined without algebraic coordinate and provides a single singularity condition which contains all generals and particulars cases. The keys elements of transition between the rows of J and singularity condition of a twist-partitioned 6-R SM are the introduction of the symbolic approach of Plücker coordinate lines and superbracket. The vanishing points of the superbracket are analyzed to describe the singularity condition. The result indicates that for a wrist-partitioned 6-R SM a single singularity condition contains three generals cases such as the shoulder, elbow and wrist singularity. Since the three last axis of the wrist are no-coplanar and intersecting at a unique point, it is suggested that for a wrist-partitioned 6-R serial manipulator, the wrist singularity never occurs physically, except that its design will be modified.
\end{abstract}

Index Terms: Singularity, 6-R Serial Manipulators, Grassmann-Cayley Algebra, Projective space, Twist graph

\section{INTRODUCTION}

Serial robot's singularity is commonly defined as configuration from which certain direction of motion may be unattainable. This defeat is due to the lost of one or more degree of twist system (TS) freedom. One approach to solve this difficulty is determining where the Jacobian matrix $J_{t}$ is non-singular for all values of the joint positions. Usually the large expressions of the determinant complicated efficient geometric analysis. To overcome this problem, several approaches have been proposed over the past years. Among these approaches Grassmann-Cayley Algebra $(G C A)$ is probably one of the most efficient since it provides sufficient tools to properly analyze geometrically the singularity condition without coordinate expression. $G C A$ approach is suitable for analyzing the rigidity of the framework of the architecture and for scene analysis [1-8].It has a powerful tools for geometric interpretation of coordinate free representation and singularity analyzing in real time computing .The solution provided in $G C A$ language by vanishing the superbrackets decomposition is a single condition which contains all general and particular cases [1-17].To prevent the clash of serial robot's actuators which are in singularity configuration, we firstly determined $J_{t}$ related to its twist and secondly calculate the dependency condition of the $\operatorname{det}\left(J_{t}\right)$ which rows are Plücker coordinate lines.
Indeed, R. Paul and C. Stevenson [18] were the pioneers to observe serial singularity from Jacobian matrix. D. Gottlieb [19] proved their observation while D. Baker [20] used topological argument to confirm them. Inter alia method, analytical approach by dividing the Jacobian matrix in four distinct square matrix[21],identification method by developing equation on the sphere [22], algebraic based on screw theory and it reciprocal $[23,25,30]$,forward and inverse kinematic equation approach [24,31,32,34,35], singularity isolation or removing the degenerate part method [26,36], geometric classification method [27-29], quaternion based on geometry algebra [38,39],constraint and actuated force method [33]etc ...

Although, singular configuration of serial robot could be detected by software simulation [37], the large expressions $\operatorname{of} \operatorname{det}\left(J_{t}\right)$ is still a challenge for researchers. In this scope $\mathrm{J}$. Merlet [40] confirmed the above-mentioned problem due its complexity .This research focuses on the singularity condition which is vanishing condition of $\operatorname{det}\left(J_{t}\right)$ using $G C A$.This method direct to singularity analysis without algebraic coordinates and makes a geometric interpretation.

for the vanishing points of the superbracket which is similar to $\operatorname{det}\left(J_{t}\right)$.

In this paper, the singularity condition for wristpartitioned 6-R serial manipulator based on the 
degeneracy of a twist system using the dependency of Plücker coordinate lines is performed in coordinate free expression to obtain a single singularity condition including all general and particular poses. The work is organized as follow: Sect.2recalls some prerequisites in projective space used in $G C A$ such as Plücker coordinate lines, twist system and it associated graph, the concept of join and meet operators. In Sect.3, describes $6-R$ wrist-partitioned $S M S$ and the adopted representation of the mechanism. Sect.4 shows the twist system graph while Sect.5 focuses on the singularity analysis of $6-R$ serial manipulator. Finally conclusion is given at Sect.6.

\section{FUNDAMENTALS CONCEPT IN} PROJECTIVE SPACE AND GCA

In this part we will give a brief background of projective geometry tools used in $G C A$ for serial robot .More details on Plücker lines and $G C A$ can be found in[4-8;15;41].

\subsection{Twist System Projective Space}

\section{Projective space}

Projective space $Q$ is commonly referred as Euclidean space added with the plane, $\Pi_{\infty}$ at infinity namely infinite plane, which is formed by the points and lines at infinity. Any pair of parallel lines in $Q$ intersects each over at a unique point at infinity while any pair of parallel planes in $Q$ intersects each over in the same and unique line at infinity.

\section{Homogeneous coordinates and Plücker coordinates lines}

In Cartesian coordinate system $R^{n}$, each point $X \in R^{n}$ is written as $X\left(x_{1}, x_{2}, \ldots, x_{n}\right)^{T}$.Adding the $(n+1) t h$ coordinate equal to 1 , we get the homogeneous coordinates of $X$.

If $t \neq 0$; the homogeneous form of $X$ is:

$$
t\left(x_{1}, x_{2}, \ldots, x_{n}, 1\right)^{T}=\left(t x_{1}, t x_{2}, \ldots, t x_{n}, t\right)^{T}
$$

Then for any vector with $(n+1)$ real number where the last coordinate is a scalar $t \neq 0$, we can recover the Cartesian coordinate of such vector by dividing it to $t$. Let accept now the $(n+1)$ th coordinate equal to 0 if and only if the point $X$ is at infinity. Toms [42] recalled that all algebraic representation of projective geometry must be homogeneous. Lilian [43] and Roy [44] confirmed respectively that any projective point (line) has four (six) homogeneous coordinates in $Q$ and each line in $Q$ is called Plücker line.

$n$ number of independents kinematic chains form (n)screw space which is a screw subspace who is composed of $(n)$ system screw .An instantaneous screw axis is a Plücker coordinate vector line with its associated pitch in a given position, then the screw axis of any robot is described as:

$$
\hat{\$}=\left[s,\left(S_{0} \times s+h s\right)\right]
$$

with, $s$ the unit vector along the screw axis, $S_{0}$ the position vector of a point on the screw axis with respect to a reference frame, $h$ the pitch of the screw.

A twist of zero pitch screw,$\hat{\$}_{0}=\left[s,\left(S_{0} \times s\right)\right]$, is a Plücker coordinate vector of a finite line which is identified as an instantaneous pure rotation of a rigid body about the axis $l$.

\subsection{Introduction to Grassmann-Cayley Algebra}

$G C A$ is a symbolic approach to Plücker coordinates. Points are represented with their homogeneous coordinates, while lines and planes are represented with their Plücker coordinates. Extensors are symbolically denoted by Plücker coordinates of lines and characterized by their step. For example in the four-dimensional vector space $V^{4}$ associated with the three-dimensional projective space $Q^{3}$, extensors of step 1,2 and 3 represent point, line $l$ and plane $P$ respectively.(See Fig-1:I,II,III).

$$
\begin{aligned}
& l=a \vee b, P=a \vee b \vee c \\
& P \wedge l^{\prime}=c
\end{aligned}
$$

They are also associated with subspaces of $V^{4}$, of dimension 1, 2 and 3 , respectively. Let $V^{n}$ be $(n)$ dimensional vector space over the field $R, W$ be $(k)$ dimensional subspace of $V^{n}$ with $\left\{w_{1}, w_{2}, \ldots, w_{k}\right\}$ a basis of $W$ and $P$ a Plücker coordinate vector of $W$.

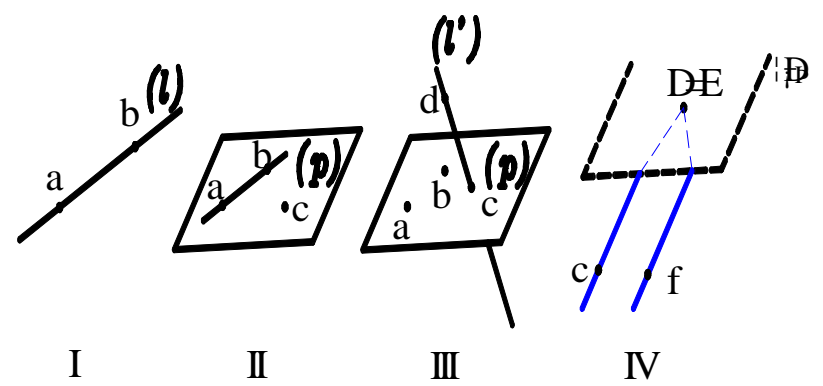

Fig-1: Illustrations of joint and meet operators and intersecting parallel lines at infinity

Consider a symbolic level in which $P$ is described without specific coordinate basis, this symbolic approach represents also a Plücker coordinate vector $P$ in the

$\mathrm{O}_{n}^{k}$-dimensional vector space of $V^{k}$ and can be written as:

$$
P=w_{1} \vee w_{2} \vee \ldots \vee w_{k}=w_{1} w_{2} \ldots w_{k}
$$

In coordinate free expression $P$ is called an extensor of step $k$. Let be $S$ a finite set of points $\left\{w_{1}, w_{2}, \ldots, w_{k}\right\}$ defined now in the $(k-1)$ dimensional Projective 
space, $Q$ over the field $R$ where $w_{i}=\left(x_{1 i}, x_{2 i}, \ldots, x_{k i}\right)^{T}$. Each $w_{i}$ is represented by it homogeneous coordinate form $k$-tuple with $1 \leq i \leq k$.The bracket of these vectors is defined as the determinant of a matrix having $w_{i}$ as its columns:

$$
\left[w_{1}, w_{2}, \ldots, w_{k}\right]=\left|\begin{array}{cccc}
x_{1,1} & x_{1,2} & \cdots & x_{1, k} \\
\vdots & \vdots & \cdots & \vdots \\
x_{k, 1} & x_{k, 2} & \cdots & x_{k, k}
\end{array}\right|
$$

In $G C A$ language this determinant formed by the homogeneous coordinate namely superbracket represents symbolically the Plücker coordinate lines .The dependency conditions of these Plücker coordinate lines is related to the vanishing conditions of the determinant .This approach is used to analyze the instantaneous kinematics as well as determining geometrically the singularity configurations in coordinate free manners. Instantaneously a twist space of serial combination of motion constraints is the sum of the all twist spaces of the composing constraints.

In GCA language a twist space of the serial connection of kinematic chains is the support of the join of all the extensors which represent the twist spaces of the chains. It provided that their twists extensors are linearly independent [41].Consequently Plücker coordinate vectors of all the system can be denoted symbolically without noting its coordinates. Eq. (4) is used to find the dependency condition. Thus, a singularity occurs when these Plücker coordinate vectors are dependent, which is equivalent to superbrackets equal to zero.

If the Plücker coordinate lines are linearly independent

$$
P=w_{1} \vee w_{2} \vee \ldots \vee w_{k} \neq 0
$$

If the Plücker coordinates lines are linearly dependent, the superbracket which is the determinant formed by the homogeneous coordinate lines vanish:

$$
\begin{aligned}
& P=w_{1} \vee w_{2} \vee \ldots \vee w_{k}=0 \\
& {\left[w_{1}, w_{2}, \ldots, w_{k}\right]=\left|\begin{array}{cccc}
x_{1,1} & x_{1,2} & \cdots & x_{1, k} \\
\vdots & \vdots & \cdots & \vdots \\
x_{k, 1} & x_{k, 2} & \cdots & x_{k, k}
\end{array}\right|=0}
\end{aligned}
$$

\section{DESCRIPTION AND ADOPTED}

\section{REPRESENTATION OF A GENERAL WRIST-}

\section{PARTITIONED 6R SERIAL MANIPULATORS}

\subsection{Description of Wrist-Partitioned 6-R SMs}

The manipulator under consideration consists of seven links $l_{i}(i=1,2, \ldots, 7)$ connected in succession by six revolute joints. The link $l_{1}$ fixed to the ground in a fixe frame $O\left(x_{1} y_{1} z_{1}\right)$ while the last three non-coplanar axis intersect at a unique common point $h$ (see Fig-2).

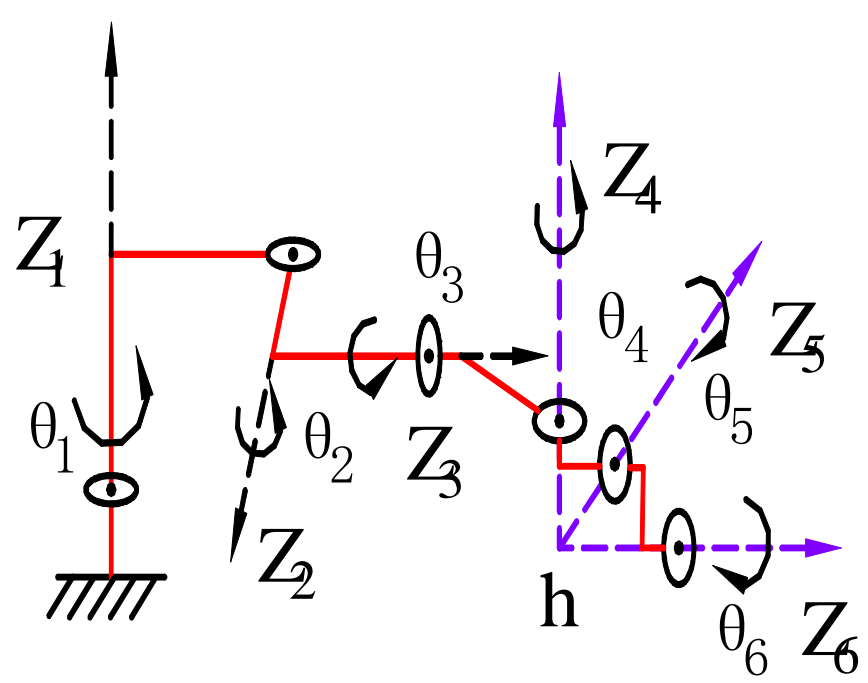

Fig-2: A general 6-R wrist-partitioned SMs

\subsection{Adopted Representations of 6-R Wrist-}

\section{Partitioned SMs}

(a) The revolute axis $z_{i}$ are respectively the joint axis of $l_{i}$ and $l_{i+1}$ where $i=1,2, \ldots, 6$

(b) $\mathrm{z}_{1}$ is orthogonal to $z_{2}$ and orthogonal to $z_{3}$.

(c) $z_{2}$ and $z_{3}$ are parallel.

(d) $z_{4}, z_{5}$ and $z_{6}$ are three no-coplanar intersecting axis at a unique point $h$.

We choose two points on each of these six joint axis centers: $a B, c D, \mathrm{fE}, \mathrm{hG}, \mathrm{hJ}$ and $h L$ respectively on $z_{1}, z_{2}, z_{3}, z_{4}, z_{5}$ and $z_{6}$ (see Fig-3)

\section{TWIST SYSTEM AND IT'S ASSOCIATED GRAPH}

\subsection{Twist System using the Symbolic Approach of}

\section{Plücker Coordinate Line in GCA Language}

Since 6-R SM consists of 6 serial joints with six center of the motion in projective space (see Fig-3.), the symbolic approach of $G C A$ which represent the Plücker coordinate vectors in coordinate free manners is the twist space $T$ of serial connection of kinematic links .It is identified as the join of all the 6-extensors, see Eq.(4), and describe as :

$$
\begin{aligned}
T & =z_{1} \vee z_{2} \vee z_{3} \vee_{4} z_{5} \vee z_{6} \\
T & =\left[z_{1} z_{2} z_{3} z_{4} z_{5} z_{6}\right]
\end{aligned}
$$




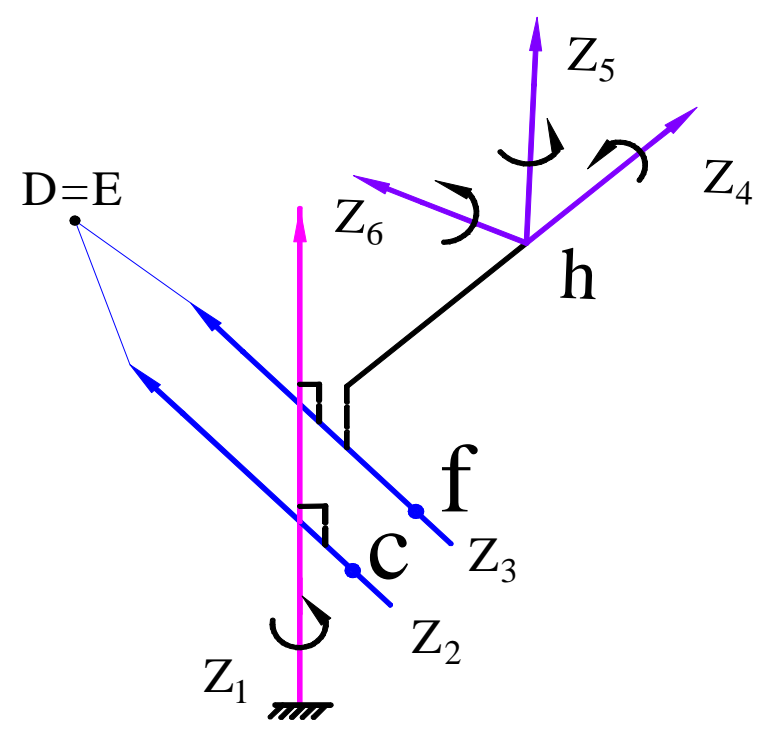

Fig-3: Adopted representation of the axis

\subsection{Twist Graph of Wrist-Partitioned 6-R SM}

The Twist graph is the graphic representation of the $T S$ in projective space. Each line is represented by one finite point and one point at infinity. The capital letters represent the points at infinity. $z_{2}$ and $z_{3}$ are parallel and intersect each over on $E=D$ at infinity. See Fig-1(IV) and Fig-4. Eq. (8) becomes:

$$
T=a B \vee c D \vee f D \vee h G \vee h J \vee h L
$$

The join of these six vectors axis in projective space represent $\operatorname{det}\left(J_{t}\right)$ which rows are the six Plücker coordinate lines and similar to the superbracket:

$$
T=[a B, c D, f D, h G, h J, h L]
$$

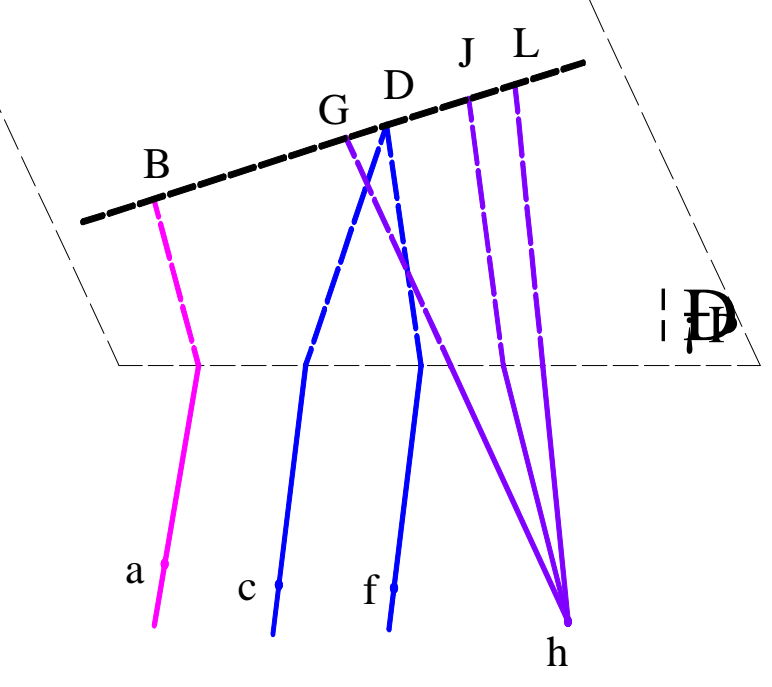

Fig-4: Twist graph of wrist partitioned 6-R SMs

\section{SINGULARITY ANALYSIS OF WRIST- PARTTIONED 6-R SM USING GCA}

The Serial singularity occurs when the robot loses one or more degree of $T S$ freedom, then the superbracket equal to zero.

\subsection{Singularity Conditions and Associated Motion of Wrist-Partitioned 6-R SM}

Geometrically singularity arises when the joint screws which are Plücker coordinate lines are linearly dependent. $\operatorname{det}\left(J_{t}\right)$ Vanishes then Eq.(10) becomes :

$$
\begin{aligned}
& T=0 \\
& {[a B, c D, f D, h G, h J, h L]=0}
\end{aligned}
$$

This expression can be developed into 24combination of linear monomials. Each of them represents the product for three brackets of four projective points $[1,3]$.The useful tool, Graphic User Interface, provided by Stéphane Caro et al [17] perform this calculus and gives the singularity condition:

$$
\begin{aligned}
& {[a B, c D, f D, h G, h J, h L]=} \\
& -[a B D h][c f D h][G j h L]
\end{aligned}
$$

And then Eq. (12) vanishes when at least one of the three monomials vanish.

\subsection{Interpretation of Singularity Condition in}

\section{Grassmann-Cayley Algebra Language}

If $[a B D h]=0$ then: $a B \wedge h D$ means $h$ lies either on $a B$ or $a B D$.According to the adopted representation $h$ lies on one of: $\left\{z_{1},\left(z_{1} z_{2}\right),\left(z_{1} z_{3}\right)\right\}$ with a particular case $h \in z_{1}$ : a shoulder singularity.

If $[c f D h]=0$ then: $c D \wedge h f$ or $f D \wedge h c$

means $h$ is either collinear to $c D$ or $f D$ since $z_{2}$ and $z_{3}$ are parallel lines and intersect in the projective space at infinity at point $D, h$ must lies on a plane formed by $z_{2} z_{3}$ : it is a an elbow singularity, the end effector is at the boundary of the workspace.

And at last if $[G J h L]=0$ then $h G \wedge L J$ or $h J \wedge L G$ or $h L \wedge G J$, means $z_{4} \wedge L J$ or $z_{5} \wedge L G$ or $z_{6} \wedge G J$, it is obvious that we are in wrist singularity condition and the last three axes $z_{4}, z_{5}, z_{6}$ must be parallel to each over: This condition is impossible to implement practically: except that the wrist -partitioned design was modified. Two of the last three axis must be parallel to each over.

\section{CONCLUSIONS}

This research presented the singularity configuration of wrist-partitioned 6-R serial manipulator based on GCA. We 
formulated the twist system by symbolic approach of Plücker coordinate lines and used an alternative coordinate free method, GCA, to detect the singularity condition. The twist graph was obtained by introducing the notion of join and meet operators. Since GCA provided sufficient tools to analyze singularity condition without algebraic coordinate, this method was implemented in this paper and the result provided a single singularity condition which contained three classes of singularity such as shoulder, elbow and wrist singularity. The analysis of these results suggested that the wrist singularity never occurs physically. Since the conceptual design of the wrist has last three axes noncoplanar and intersecting at a unique point, it was deduced that the wrist singularity could be possible if and only if the design of two of the last three axes are parallel.

However, we recognize the current work was not entirely absolute due to the complexity of the topic. The use of GCA approach to analyze singularity of such robot without algebraic coordinate was our contribution. These results may motivate further researchers toward GCA to analysis singularity condition of any serial robot. Elsewhere the great powerful tools used in GCA language are also useful in robotics vision or image modeling.

\section{ACKNOWLEDGEMENT}

I wish to thank Doctor ADOKO AMOUSSOU Koffi for his inspiring advice, support and encouragement during my research. I'm grateful to the editor and reviewers for their support.

\section{REFERENCES}

[1]. P.Ben-Horin, M.Shoham. Singularity Analysis of a Class of Parallel Robots based on Grassmann-Cayley Algebra. Mechanism and Machine Theory, 41, no.8 (2006), 958-970.

[2]. P.Ben-Horin, M.Shoham.Singularity Condition of SixDegree-of-Freedom Three-Legged Parallel Robots Based on Grassmann-Cayley Algebra. IEEE Transactions on Robotics, 22, no.4 (2006), 577-590.

[3]. P.Ben-Horin,M. Shoham.Application of Grassmann Cayley Algebra to Geometrical Interpretation of Parallel Robot Singularities. The International Journal of Robotics Research, 28, no.1 (2009), 127-141.

[4]. Laurent Fuchs. Algèbre de Grassmann-Cayley. XLIMSIC, Université de Poitiers, France INRIA Sophia-Antipolis (2009)1-13.

[5]. Neil L . White. A Tutorial on Grassmann-Cayley Algebra, in:Invariant Methods in Discrete and Computational Geometry, Proceedings of the Curaçao Conference. Springer Netherlands (1994) pp 93-106.

[6].Neil L. White. Grassmann-Cayley Algebra and Robotics Applications, in: Handbook of Geometric Computing. Applications in Pattern Recognition,Computer Vision, Neural computing, and Robotics, 8, Springer-Verlag Berlin Heidelberg (2005), pp 629 -656.

[7]. Neil L. White.Grassmann-Cayley Algebra and Robotics. Journal of Intelligent and Robotic Systems, 11,(1994)91107.
[8]. E. Staffetti, F.Thomas. Kinestatic Analysis of Serial and Parallel Robot Manipulators Using Grassman-Cayley Algebra, in: J.Lenarcic and M.M. Stanisic (eds.), Advances in robot Kinematics, Springer Netherlands (2000), pp17-27. [9]. S.Caro, G.Moroz , T.Gayral ,D.Chablat, C. Chen. Singularity Analysis of a Six-Dof Parallel Manipulator Using Grassmann-Cayley Algebra and Gröbner Bases . J. Angeles et al. (Eds.) Brain, Body and Machine. Advances in Intelligent and Soft Computing,83, Springer Berlin Heidelberg (2010),pp341-352.

[10]. Laurent Fuchs and Laurent Théry. A Formalization of Grassmann-Cayley Algebra in Coq and its Application to Theorem Proving in Projective Geometry, in : Automated Deduction in Geometry .Lecture Notes in ComputerScience,6877 ,Springer Berlin Heidelberg (2011), pp 51-67.

[11].D.Kanaan, P.Wenger ,D.Chablat. Singularity Analysis of Limited-DOF Parallel Manipulators Using GrassmannCayley Algebra. Advances in Robot Kinematics: Analysis and Design. Springer Netherlands(2008),pp 59-68.

[12]. D.Kanaan, P. Wenger, S.Caro, D.Chablat. Singularity Analysis of Lower Mobility Parallel Manipulators Using Grassmann-Cayley Algebra. IEEE Transactions on Robotics, 25, no.5 (2009) 995-1004.

[13]. S.Amine, M.T.Masouleh, S.Caro , P.Wenger, C.Gosselin. Singularity Conditions of 3T1R Parallel Manipulators with Identical Limb Structures . ASME ,Journal of Mechanisms and Robotics, 4, 1 (2012).

[14]. S. Amine, M.T.Masouleh, S. Caro,P.Wenger, C.Gosselin. Singularity analysis of 3T2R parallel mechanisms using Grassmann-Cayley Algebra and Grassmann geometry. Mechanism and Machine Theory ,52 ,(2012), 326-340.

[15].Hongbo Li. From Past to Future: Graßmann's Work in Context. Projective Geometric Theorem Proving with Grassmann-Cayley Algebra. Graßmann Bicentennial Conference,September 2009 Springer Basel (2011) pp 275285.

[16]. Lennart RUBBERT .Conception de Mécanismes Compliants pour la Robotique Chirurgicale. Ph-D Dissertation .Université de Strasbourg.(2012),pp96.

[17]. S.Amine ,S.Caro.Singularity Conditions of LowerMobility Parallel Manipulators Based on Grassmann-Cayley Algebra. L'Agence nationale de la recherche. Retrieved December 1, 2013, from http ://www.irccyn.ecnantes.fr/ caro/SIROPA/GUIGCASiropa.jar

[18]. R.P. Paul, C.N. Stevenson. Kinematics of Robot Wrists .The International Journal of Robotics Research, 2, no.1,(1983)31-38.

[19]. D.H. Gottlieb. Robots and Fibre Bundles. Bull. Soc. Math. Belg. Sér. A, 38, (1987) 219-223.

[20]. D. R. Baker , C.W.Wampler II .The International Journal of Robotics Research, 7 ,no. 2,(1988 ) 3-21.

[21]. M. J. D. Hayes, M. L. Husty, P. J. Zsombor-Murray. Singular configurations of wrist-partitioned 6R serial robots: a geometric perspective for users. Transactions of the Canadian Society for Mechanical Engineering, 26, no. 1 (2002) 41-55.

[22]. Z.C. Lai, D.C.H. Yang. A New Method for the Singularity Analysis of Simple Six-link Manipulators. The 
International Journal of Robotics Research,5, no.2 (1986) 66-74.

[23]. Y-C. Chen ,C.L. P.Chen .An Analysis to the Singularity of Serial Manipulator using reciprocal screw theory. IEEE International Conference on: Systems, Man, and Cybernetics, Humans, Information and Technology, 1, (1994)148 - 153.

[24]. Y.C. Huang, D. K. Qu, F. Xu, W. X. Zhang. An Approach Dealing with Wrist Singularity of Six-DOF Industrial Robots. Advanced Materials Research, 490-495, (2012)1936-1940.

[25].Man Bok Hong .On the Robot Singularity: A Novel Geometric Approach. International Journal of Advanced Robotic Systems, 9, (2012)197-208.

[26]. F-T. Cheng, T-L. Hour, Y-Y.Sun,T-H. Chen. Study and Resolution of Singularities for a 6-DOF PUMA Manipulator. IEEE Transactions on: Systems, Man, and Cybernetics-Part B: Cybernetic, 27, no. 2, (1997)332-343.

[27]. M. Husty, E. Ottaviano ,M. Ceccarelli . Advances in Robot Kinematics: Analysis and Design .A Geometrical Characterization of Workspace Singularities in $3 R$ Manipulators. Springer Netherlands (2008),pp 411-418.

[28]. Krzysztof Tchoń. Singularity of Euler Wrist. Mechanism and Machine Theory, 35, (2000) 505-515.

[29]. Shiva Shankar. Singularities Mechanisms II-trajectory tracking in the 3-axis wrist. Mechanism and Machine Theory, 34, (1999) 513-526.

[30]. R.Boudreau, R.P. Podhorodeski.Singularity Analysis of a Kinematically Simple Class of 7 Jointed Revolute Manipulators. Transactions of the Canadian Society for Mechanical Engineering,34,1, (2010)105-117.

[31]. M. Vaezi , F.C. Samavati , H.E.S.Jazeh , S.A. A.Moosavian .Singularity Analysis of 6 DOF Stäubli ${ }^{\circledR}$ TX40 Robot. IEEE International Conference on Mechatronics and Automation (2011)446-541.

[32]. H. Liu, W. Zhou, X.Lai,S. Zhu. An Efficient Inverse Kinematic Algorithm for a Puma560 -Structured Robot Manipulator. International Journal of Advanced Robotic Systems, 10, (2013) $236-240$.

[33]. S.J. Remis ,M. M. Stanišić .Design of a SingularityFree Articulated Arm Subassembly. IEEE Transactions on Robotics and Automaton, 9, no6, (1993)816-824.

[34]. K-S. Chang ,O. Khatib .Manipulator Control at Kinematic Singularities: A Dynamically Consistent Strategy. IEEE/RSJ International Conference on Intelligent Robots and Systems . 'Human Robot Interaction and Cooperative Robots', Proceedings,3,(1995)84-88.

[35]. K.Tchoń ,R. Muszysńki. Singular Inverse Kinematic Problem for Robotic Manipulators: A Normal Form Approach. IEEE Transactions on Robotics and Automation, 14, no.1, (1998)93-104.

[36]. D.Oetomo, M.Ang Jr. , Ser Y. Lim .Singularity Handling on Puma in Operational Space Formulation. Experimental RoboticsVII. Lecture Notes in Control and Information Sciences, 271, Springer Berlin Heidelberg (2001) pp 491-500.

[37]. Adept Technology, Inc. Six-Axis Robot Configuration Singularities. Use of the V+ MVSL-Move Routine and the Speed Limit Parameter . Intelligent Robotics for the Global Economy .Retrieved December 1, 2007, from http://www1.adept.com/main/ke/data/procedures/singularity /singularity.pdf

[38].A. Stolt, M.Linderoth, A.Robertsson, R.Johansson. Robotic Assembly Using a Singularity-Free Orientation Representation Based on Quaternions. 10th International IFAC Symposium on Robot Control, 10, Part.1 (2012) 549554.

[39]. E.Sariyildiz, H.Temeltas. A new formulation method for solving kinematic problems of multiarm robot systems using quaternion algebra in the screw theory framework.Turkish Journal of Electrical Engineering \& Computer Sciences,20,no.4,(2012) 607- 628.

[40]. J-P.Merlet. Parallel Robots -2e Edition. Chap6: Singular Configuration .Solid Mechanics and Its Applications . Springer Netherlands, (2006) pp 185.

[41]. Neil L White. Multilinear Cayley Factorization. Journal of Symbolic Computation, 11, no.5-6, (1991), 421438.

[42]. Tom Davis. Projective Geometry. Mathematical Circles Topics, (2001). Retrieved December 1, 2013, from http://www.geometer.org/mathcircles/projective.pdf [43]. Lilian Aveneau.Les Coordonnées de Plücker Revisitées. Revue Électronique Francophone d'Informatique Graphique, 3, no.2, (2009)59-68.

[44]. Roy Featherstone. Plücker Basis Vectors. Proceedings IEEE International Conference on Robotics and Automation, Orlando Fl, (2006) 1892-1897

\section{BIOGRAPHY}

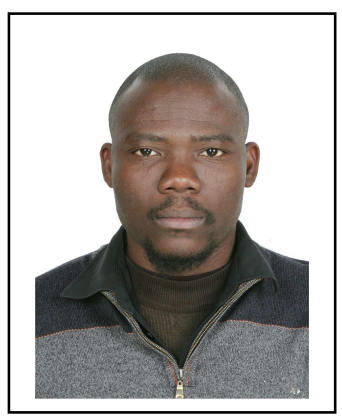

AKONDE L. D.Clément. Currently $\mathrm{PhD}$ Student in Mechatronics at School of Mechanical Science and Engineering .Huazhong University of Science and Technology (Wuhan-China).He received Master in Mechatronics at School of Mechanical Science and Engineering of Huazhong University of Science and Technology. Research fields: Mechanic of Robot and Mechanism of Robot Manipulators. Mathematic of Robot Manipulation 